\title{
Regional Anaesthesia, The Seed of Opportunity In COVID-19 Crisis: The Balance Has Shifted
}

\author{
Shiv Kumar Singh ${ }^{1}$, Maria Alexandra Tsoutsou ${ }^{1}$
}

Albert Einstein once said, "in the midst of every crisis, lies great opportunity”. There's no question that we're in the midst of a global crisis. There's no doubt that a crisis creates problems, lots of them, but it also creates opportunities. Something that every anaesthetist does day in day out safely, intubation of trachea, is now become a risk factor for spread of the disease.

So where is the opportunity in this crisis? In the west, regional anaesthesia is often used as an adjunct rather than as sole anaesthetic technique, as part of multimodal analgesia in patients who are being operated under general anaesthesia. Unfortunately, general anaesthesia requires airway manipulation that is associated with aerosol generation and risks transmission of corona virus. This is a risk that can be averted with use of regional anaesthesia techniques for procedures that can be done with patient awake rather than asleep.

At the beginning of the pandemic with surge of patients requiring endotracheal intubation and ventilation, increased intensive care admissions affected anaesthesia services in many ways. The increased number of patients needing critical care increased the demand for drugs used in both anaesthesia and critical care and this demand led to shortage of anaesthesia drugs and led the Association of Anaesthetists (AOA) and the Royal College of Anaesthetists (RCoA), working closely with the Chief Pharmaceutical Officer at NHS England to produce a guidance which summarised potential mitigations to be used in the management of such demand. Direct alternative drugs and techniques were offered [1]. The options identified in the guidelines were not exhaustive but give a way of thinking about this situation we all have landed up in. We were unsure of how long this demand would continue and how we would manage the situation. This is where the opportunity to use regional anaesthesia for procedures that could be done purely under neuraxial or peripheral nerve blocks became a necessity. With just a few mls of local anaesthesia we could perform the whole surgeries. We could not only save on the anaesthetic drugs; we also provided a safer environment with much less risk of contracting the virus that had frightened most out of our guts.

Both the American Society of Regional Anaesthesia (ASRA) and European Society of Regional Anaesthesia and Pain Therapy (ESRA) came up with joint guidelines very early on during the pandemic on utilisation of neuraxial anaesthesia and peripheral nerve blocks [2]. These guidelines described various practical aspects with regard to perioperative management of patients with COVID-19 using RA techniques. Both societies recommended wider adoption of regional anaesthesia techniques by the anaesthetists/anesthesiologist to protect themselves from additional exposure to novel coronavirus and hoped that acceptance of this new norm would help in improving patient safety and perioperative management.

These new guidelines exposed the lacunae in regional anaesthesia training esp peripheral nerve blocks amongst the anaesthetists. The anaesthetists could no longer cover up their partial or failed blocks with general anaesthesia, you needed to be able to demonstrate proficiency in regional anaesthesia techniques such that you had a fail-safe block without the need to use sedation or conversion to general anaesthesia.

This was the time to shun the egos and use the service of those who have the proficiency and skills in nerve blocks at the same time use the opportunity to hone new skills. This was a great time to learn and improve our skills and knowledge about regional anaesthesia. Both regional anaesthesia societies also suggest use of "block teams" similar to "intubation teams". The idea was to use the expertise of those who are proficient in the field of anaesthesia and thus avoid failures of block and conversions to general anaesthesia. These experts could supervise those who wanted to refine their regional anaesthesia techniques, an opportunity that COVID-19 had provided.

We had stopped all routine surgeries where the blocks are normally be utilised esp routine orthopaedic surgeries, but we still had a steady stream of high-risk cases that need operations on their limbs due to vascular insufficiency and diabetes related complications. Then there were the trauma cases where regional anaesthesia techniques are often utilised routinely. We have many examples where we have used regional anaesthesia for high-risk vascular cases and averted admissions to high dependency units. In this issue we present one such interesting

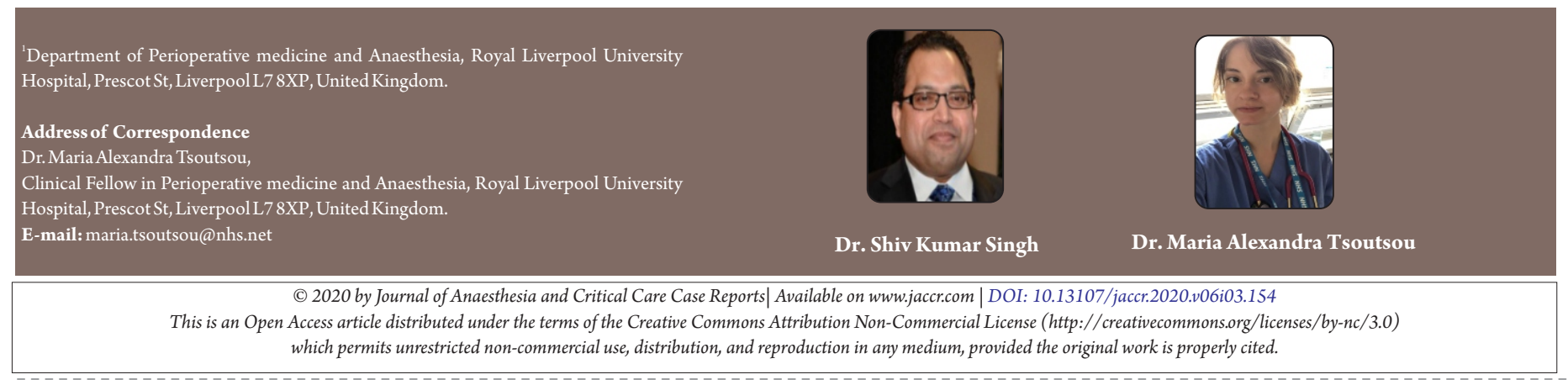

03 | Journal of Anaesthesia and Critical Care Case Reports | Volume 6; Issue 3 | September-December 2020| Page 03-04 
case that had to be admitted to HDU for few days following his previous surgery and now presented to us for another emergency procedure due to failure of the previous surgery. We utilised regional anaesthesia technique and patient could be discharged directly to the ward [3].

Kopacz, Neal and Pollock in their 1996 publication, the regional anaesthesia 'learning curve' suggested that approximately 20-25 procedures each are necessary before improvement in the techniques of spinal and epidural anaesthesia is demonstrated by residents in training. For a $90 \%$ success rate, 45 and 60 attempts at spinal and epidural anaesthesia, respectively, are necessary [4]. These figures are lacking for peripheral nerve blocks that require greater skills and knowledge to be able to visualise the nerve and be able to deposit local anaesthetic in the right place for it to be effective in order to conduct surgery solely under regional anaesthesia technique. It further needs knowledge of surgical anatomy to consider the dermatomes, myotomes and for orthopaedics procedures, the osteotomes that are involved. Knowledge about how to assess the efficacy of the blocks and in case of sparing/failure without resorting to conversion to general anaesthesia, the ability to administer rescue blocks in such situations is essential to provide this kind of service.
In later publication in year 2000, Kopacz and Neal concluded that anaesthesiology training programs in US now appear to provide a satisfactory exposure to regional anaesthesia for a majority of resident trainees, although $40 \%$ of residents may still be deficient in nerve block anaesthesia. The growth in the use of regional anaesthesia during residency has plateaued over the past decade, but the discrepancy between individual resident experience has improved [5]. This data is now 20 years old but we still observe similar trends. Data from UK training program in regional anaesthesia is lacking. Such data would require a robust database system and we have mentioned how this can be done using RAD App [6].

Hadzic, Vloka and Koenigsamen in their publication in 2002 note that a structured regional anaesthesia rotation, a dedicated team of mentors with training in regional anaesthesia, and adequate clinical volume are a pre-requisite for adequate training, but they may not be available in many anaesthesia residency training programs [7]. They further suggest that as the demand for regional anaesthesia continues to increase, it is imperative to ensure adequate education/training of anaesthesia residents to meet this demand. In order to achieve this goal, they suggest that the recommendations should be re-evaluated, and perhaps a proficiency in a core group of widely applicable and relatively simple nerve blocks should be mastered by all graduates. We totally agree with this observation as mastery over certain common blocks like femoral and popliteal sciatic nerve blocks for the lower limb, interscalene and supraclavicular blocks for the upper limb could solve many of the issues while acquiring competency in regional anaesthesia. Proficiency in other complex blocks can be developed later during their anaesthesia career.

To conclude, COVID-19 has presented the opportunity for consultants and trainees to observe, learn and refine their skills at administration of peripheral nerve blocks under the guidance of those who have expertise in the field of regional anaesthesia and this opportunity should not be missed. Every crisis presents us with opportunities, and it is up to us how we use these opportunities to teach and learn. These are times where people need to set aside their fears and egos and hone newer skills that can also be utilised in the present times, as well as the better times we all look forward to.

\section{References}

1. Guidance on potential changes to anaesthetic drug usage and administration during pandemic emergency pressures ICM Anaesthesia COVID-19. [cited 2020 Nov 26]. Available from: https://icmanaesthesiacovid-19.org/drug-demand-supply-anaesthetic-drug-usage-andadministration

2. Uppal $V$, Sondekoppam RV, Lobo CA, et al. Practice recommendations on neuraxial anesthesia and peripheral nerve blocks during the COVID-19 Pandemic- a joint statement by the American Society of regional anesthesia and pain medicine (ASRA) and European Society of regional anesthesia and pain therapy (ESRA), March 31, 2020. Available: https:// www.asra.com/content/documents/ra-covid19_final.pdf

3. Tsoutsou MA and Singh SK, Covid-19 And Regional Anaesthesia: Use Of Ultrasound Guided Popliteal Sciatic Nerve Blocks For Emergency Forefoot Amputation In A High Risk Deaf And Mute Covid-19 Contact Patient; Journal of Anaesthesia and Critical Care Reports; 6(3), September-December 2020; Page 08-10.

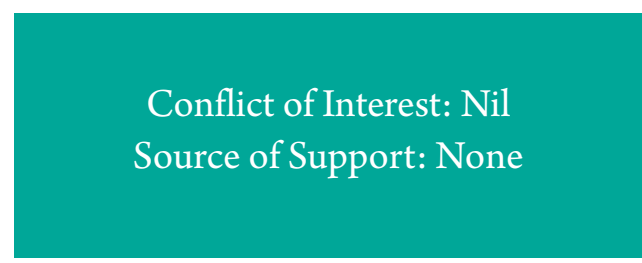

\section{How to Cite this Article}

Singh SK, Tsoutsou MA | Regional Anaesthesia, The Seed of Opportunity In COVID-19 Crisis: The Balance Has Shifted | Journal of Anaesthesia and Critical Care Case Reports | September-December 2020; 6(3): 03-04. 